\title{
Letter regarding article by Wang et al., "Internal fixation of distal tibiofibular syndesmotic injuries: a systematic review with meta-analysis"
}

\author{
Mingbo Tian • Mingsheng Zhang $•$ Xiaowei Guo $\cdot$ Shoufeng Li
}

Received: 14 August 2013 / Accepted: 1 September 2013 /Published online: 2 October 2013

(C) Springer-Verlag Berlin Heidelberg 2013

To the Editor,

We read with great interest the article by Wang et al. [1] titled

"Internal fixation of distal tibiofibular syndesmotic injuries: a systematic review with meta-analysis" published on-line in July 2013 in International Orthopaedics. In this meta-analysis, Wang et al. analysed the optimal method for syndesmotic fixation. Four comparisons with traditional metallic screw were identified in terms of bioabsorbable screws, tricortical fixation method, suture-button device and nonfixation in low syndesmotic injuries. The authors concluded that patients with low syndesmotic injuries should be well assessed before fixation determination and that the indication for screw placement in such conditions needs to be further defined. Nevertheless, we have several queries to communicate with the authors.

1. The authors have not focused specifically or in any detail on the issue of the completeness of the search strategy report for databases. They just described the retrieval strategy by using the keywords "syndesmosis" or "tibiofibular". We suggest that the authors provide details of the retrieval strategy.

2. The authors defined one exclusion criterion as "study subjects to be less than 20". We are wondering the reason for this exclusion criterion. Among the included studies, the minimum number of subjects was 24 by Coetzee et al. [2]

M. Tian $(\bowtie) \cdot$ S. Li

Department of Traumatic Orthopaedics, People's Hospital of

Zhengzhou, Zhengzhou 450047, Henan Province,

People's Republic of China

e-mail: tianmingbo450052@126.com

M. Zhang

Department of Orthopaedics, The Second Affiliated Hospital of Zhengzhou University, Zhengzhou 450014, Henan Province,

People's Republic of China

X. Guo

Department of Spinal Surgery, Zhengzhou Orthopaedics Hospital, Zhengzhou 450052, Henan Province, People's Republic of China and Pakarinen et al. [3]. Therefore, we suggest that exclusion criteria should be redefined by the authors.

3. We suggest that the authors evaluate the methodological quality of the included studies, which could avoid potential bias in the meta-analysis. Each included study should be independently assessed by two reviewers. Meanwhile, the authors also should clarify how to assess the quality of all included studies and detailed scores for each trial.

4. The authors used a fixed-effects model to pool data in all forest plots (Tables 2-5). However, different effects models may result in different results. Therefore, we would like to know the reason that the authors chose the fixed effects model for all analyses. In our opinion, the authors should pay attention to the $I^{2}$, which is on behalf of heterogeneity. When $I^{2}$ was under $50 \%$, low heterogeneity was assumed, and the fixed-effects model could be used to pool data. Conversely, when $I^{2}$ exceeded $50 \%$, heterogeneity was thought to exist and the effects model should be random.

Moreover, further high-quality randomised controlled trials (RCTs) based on larger sample sizes are still needed. We believe that our remarks will contribute to more accurate elaboration of the results presented by Wang et al.

Conflict of interest There is no conflict of interest and the paper has not been submitted elsewhere.

\section{References}

1. Wang C, Ma X, Wang X, Huang J, Zhang C, Chen L (2013) Internal fixation of distal tibiofibular syndesmotic injuries: a systematic review with meta-analysis. Int Orthop. doi:10.1007/s00264-013-1999-x

2. Coetzee JC, Ebeling P (2009) Treatment of syndesmoses disruptions: a prospective, randomized study comparing conventional screw fixation vs TightRope fiber wire fixation-medium term results. S Afr Orthop J 8(1): 32-37

3. Pakarinen HJ, Flinkkilä TE, Ohtonen PP (2011) Syndesmotic fixation in supination-external rotation ankle fractures: a prospective randomized study. Foot Ankle Int 32:1103-1109 